\title{
RIEMANN SUMS AND MODULAR FUNCTIONS ON LOCALLY COMPACT GROUPS
}

\author{
Kenneth A. Ross and George Willis
}

Dedicated to the memory of Karl Stromberg (1931-1994)

In 1967 the first author and Karl Stromberg published a theorem concerning generalized limits of Riemann sums on locally compact groups. The setting is a locally compact group $G$ and an increasing sequence $H_{n}$ of closed subgroups whose union is dense in $G$. The theorem was shown to hold provided that the restriction of the modular function on $G$ to $H_{n}$ agrees with the modular function of $H_{n}$ for all large $n$. This hypothesis holds in many cases and, in fact, Ross and Stromberg were unable to determine whether the hypothesis was really needed for the theorem or even whether this hypothesis always holds. An example is provided which shows that this hypothesis does not always hold. It is then shown that the theorem fails without the hypothesis.

In 1967 the first author and Karl Stromberg [R\&S] proved a generalization of Jessen's theorem $[\mathbf{J}]$ on Riemann sums as follows. Let $\left\{H_{n}\right\}$ be an increasing sequence of closed subgroups of a locally compact group $G$ whose union is dense in $G$. For each $n$, let $\Delta_{n}$ and $\lambda_{n}$ denote the modular function and left Haar measure on $H_{n}$. Similarly, let $\Delta$ and $\lambda$ denote the modular function and left Haar measure on $G$. Then Theorem 1 in $[\mathbf{R} \& \mathbf{S}]$ shows that the measures $\lambda_{n}$ can be normalised so that $\lim _{n} \int_{H_{n}} f d \lambda_{n}=\int_{G} f d \lambda$ for all continuous functions on $G$ with compact support. For a function $f$ on $G$ and $x$ in $G$, let ${ }_{x} f$ denote the translation defined by ${ }_{x} f(y)=f(x y)$ for $y$ in $G$.

\section{Theorem (Jessen; Ross and Stromberg). If}

$$
\text { the restriction of } \Delta \text { to } H_{n} \text { agrees with } \Delta_{n} \text { for all large } n \text {, }
$$

then for every $f$ in $L_{1}(G, \lambda)$

$$
\lim _{n} \int_{H_{n}} f d \lambda_{n}=\int_{G} f d \lambda
$$

for $\lambda$ locally almost all $x$ in $G$. 
The key tool in proving the theorem was a theorem of Edwards and Hewitt $[\mathbf{E} \& \mathbf{H}]$ on pointwise limits of sublinear operators whose ranges are families of measurable functions. One of the hypotheses of the Edwards-Hewitt theorem translated to the requirement that, for some $n_{0}, \sup _{n \geq n_{0}} \int_{H_{n}} f d \lambda_{n}<\infty$ locally almost everywhere for all non-negative integrable functions $f$ on $G$. The hypothesis $(\mathrm{P})$ was only needed in order to verify this.

Property $(\mathrm{P})$ holds if the $H_{n}$ 's are unimodular (hence if they are abelian, compact or discrete) or if the $H_{n}$ 's are open subgroups or normal subgroups. It was not determined whether hypothesis $(\mathrm{P})$ was needed in the theorem nor whether property $(\mathrm{P})$ can ever fail. The authors wrote, "It seems unlikely that $(\mathrm{P})$ must hold, but the authors unfortunately have not been able to produce an example to settle this question." After 28 years, the second author provided an example to show that $(\mathrm{P})$ can fail and the first author observed that the theorem must fail without the presence of $(\mathrm{P})$.

The example is a totally disconnected group and the next couple of paragraphs discuss property $(\mathrm{P})$ for such groups. The remainder of the paper gives the example and the explanation of why the theorem fails.

Theorem 4 in $[\mathbf{R \& S}]$ asserts that, if $F$ is a compact subset of one of the closed subgroups, $H_{m}$ say, of $G$, then

$$
\lim _{n} \Delta_{n}(x)=\Delta(x)
$$

uniformly for $x$ in $F$. In the case of totally disconnected groups a sharper conclusion can be made. It is shown in [W2] that, if $G$ is totally disconnected, there is an $n_{0} \geq m$ such that

$$
\Delta_{n}(x)=\Delta(x)
$$

for every $x$ in $F$ and every $n \geq n_{0}$. The proof makes use of the scale function of a totally disconnected group $G$, which is shown to exist in [W1]. The scale function, $s$, is a continuous function on $G$ which takes positive integer values and satisfies

$$
\Delta(x)=s(x) s\left(x^{-1}\right)^{-1}
$$

for every $x$ in $G$. It is shown in [W2] that, for each $x$ in $G$, the sequence $\left\{s_{n}(x)\right\}_{n=1}^{\infty}$ is constant after finitely many terms and that this convergence occurs uniformly for $x$ in compact subsets of $G$. This fact, together with (1) and (3), implies (2). Note that, although $s_{n}(x)$ becomes constant as $n$ increases, it does not necessarily become equal to $s(x)$; see [W2] for an example.

If $H_{m}$ is compactly generated, then (2) implies that $\left.\Delta_{n}\right|_{H_{m}}=\left.\Delta\right|_{H_{m}}$ for all sufficiently large $n$. At first glance, this seems to be very close to saying that 
the condition $(\mathrm{P})$ is satisfied. However the apparently slight gap between this statement and condition $(\mathrm{P})$ cannot be closed as the example below shows. These remarks serve to indicate what a group which fails to satisfy (P) must be like and indeed in the example, $\left.\Delta_{n+1}\right|_{H_{n}}=\left.\Delta\right|_{H_{n}}$ for all $n$ but $\left.\Delta_{n}\right|_{H_{n}} \neq\left.\Delta\right|_{H_{n}}$.

\section{The Group and Subgroups.}

In the following description of the example, $\mathbb{Z}_{2}$ denotes the group $\{0,1\}$ with addition modulo 2 . Let $N$ be the set of functions

$$
h: \mathbb{Z} \times \mathbb{N} \rightarrow \mathbb{Z}_{2}:(i, j) \mapsto h_{i j}
$$

such that $h_{i j}=1$ for only finitely many $(i, j)$ with $i \leq 0$. In other words, $h$ belongs to $N$ if there are $p$ in $\mathbb{Z}$ and $q$ in $\mathbb{N}$, depending on $h$, such that

$$
h_{i j}=0 \quad \text { if } i<p \quad \text { or if } i \leq 0 \text { and } j>q .
$$

Then $N$ becomes an abelian group when equipped with pointwise addition.

Let $K$ be the subgroup

$$
K=\left\{h \in N: h_{i j}=0 \text { if } i \leq 0\right\}
$$

and for each finite subset, $\mathcal{F}$, of $\mathbb{N} \times \mathbb{N}$ let

$$
U_{\mathcal{F}}=\left\{h \in K: h_{i j}=0 \text { if }(i, j) \in \mathcal{F}\right\} .
$$

Then $\left\{h+U_{\mathcal{F}}: h \in N ; \mathcal{F}\right.$ a finite subset of $\left.\mathbb{N} \times \mathbb{N}\right\}$ is a base of neighbourhoods for a topology on $N$ which makes $N$ a topological group. With respect to this topology, $K$ is a compact, open subgroup of $N$. $K$ is compact because it is just the infinite product of finite groups, that is, $K=\Pi_{\mathbb{N} \times \mathbb{N}} \mathbb{Z}_{2}$ with the product topology. The quotient group $N / K$ is discrete and is isomorphic to the direct sum of countably many copies of $\mathbb{Z}_{2}$.

For each $p$ in $\mathbb{N}$ define $\alpha_{p}: N \rightarrow N$ by

$$
\left(\alpha_{p}(h)\right)_{i j}=\left\{\begin{array}{ll}
h_{i+1 j}, & \text { if } j=p \\
h_{i j}, & \text { if } j \neq p
\end{array} .\right.
$$

Then $\alpha_{p}$ is a continuous automorphism of $N$ and the $\alpha_{p}$ 's commute with each other. Let $A=\oplus_{p>0} \mathbb{Z}$ be the direct sum of copies of the group of integers and denote elements of $A$ by $\mathbf{k}=\left(k_{1}, k_{2}, k_{3}, \ldots\right)$, where $k_{p}$ is an integer and $k_{p} \neq 0$ for only finitely many $p$ 's. $A$ will be equipped with the discrete topology. Define a homomorphism $\alpha: A \rightarrow \operatorname{Aut}(N)$ by

$$
\alpha\left(k_{1}, k_{2}, k_{3}, \ldots\right)=\alpha_{1}^{k_{1}} \alpha_{2}^{k_{2}} \alpha_{3}^{k_{3}} \ldots
$$


The apparently infinite product on the right hand side makes sense because only finitely many of the exponents are non-zero. This product will also be denoted $\alpha^{\mathbf{k}}$. Finally, define $G$ to be the semi-direct product

$$
G=A \times{ }_{\alpha} N
$$

The automorphisms $\alpha_{p}$ are regarded as belonging to $G$, and $N$ is regarded as being a subgroup of $G$. Then the base of neighbourhoods of the identity in $N$ described above is also a base of neighbourhoods of the identity in $G$. General elements of $G$ have the form $\alpha^{\mathbf{k}} h$ and the product of two elements $\alpha^{\mathbf{m}} g$ and $\alpha^{\mathbf{k}} h$ is

$$
\alpha^{\mathbf{m}} g \alpha^{\mathbf{k}} h=\alpha^{\mathbf{m}+\mathbf{k}}\left(\alpha^{\mathbf{k}}(g)+h\right) .
$$

For each $n \geq 1$ let $N_{n}$ be the closed subgroup of $N$

$$
N_{n}=\left\{h \in N: h_{i j}=0 \text { if } j>n\right\}
$$

and let $K_{n}=N_{n} \cap K$. Then $\bigcup_{n=1}^{\infty} K_{n}$ is dense in $K$ because $K$ has the product topology. It is also clear that $\bigcup_{n=1}^{\infty} N_{n}$ is dense in $N$. Since $K$ is an open subgroup of $N$, it follows that $N=\bigcup_{n=1}^{\infty} N_{n}+K$. Let $H_{n}$ be the closed subgroup of $G$ generated by $N_{n}$ and $\alpha_{1}, \alpha_{2}, \ldots, \alpha_{n}, \alpha_{n+1}$. Then $H=\bigcup_{n=1}^{\infty} H_{n}$ is dense in $G$. Since $K$ is an open subgroup of $G$, it follows that $G$ is equal to the increasing unions

$$
G=\bigcup_{n=1}^{\infty} H_{n} K=\bigcup_{n=1}^{\infty} K H_{n} .
$$

For each natural number $p$ we have

$$
\alpha_{p}^{-1} K \alpha_{p}=\alpha_{p}(K)=K \cup\left(h^{(p)}+K\right),
$$

where

$$
h_{i j}^{(p)}= \begin{cases}1, & \text { if } i=0 \text { and } j=p \\ 0, & \text { otherwise. }\end{cases}
$$

Hence $H_{n} K$ is not a subgroup of $G$ for any $n$. For the same reason $\alpha_{n+1}$ does not belong to $Z(G)$, the centre of $G$. Note however that, for each $n$, $\alpha_{n+1}$ does belong to $Z\left(H_{n}\right)$. This observation is the key to the example.

\section{Haar Measures and Modular Functions.}

Let $\lambda$ be the left invariant Haar measure on $G$, normalised so that $\lambda(K)=1$. Since $G$ is the disjoint union of $K$-cosets, in order to describe the Haar measure on $G$ it suffices to describe its restriction to $K$. Since $K$ is an 
infinite product of copies of $\mathbb{Z}_{2}=\{0,1\}$, its invariant measure is the product of copies of the measure $\frac{1}{2} \delta_{0}+\frac{1}{2} \delta_{1}$ on $\mathbb{Z}_{2}$.

The modular function on $G$ is given by $\Delta(x)=\lambda(K x) / \lambda(K)=\lambda\left(x^{-1} K x\right)$. Since $N$ is abelian and $K$ is a subgroup of $N, \Delta(h)=1$ for every $h$ in $N$. It follows from (5) that, for each natural number $p, \Delta\left(\alpha_{p}\right)=\lambda\left(\alpha_{p}^{-1} K \alpha_{p}\right)=$ $\lambda(K)+\lambda\left(h^{(p)}+K\right)=2$. For a general element of $G$ we therefore have

$$
\Delta\left(\alpha^{\mathbf{k}} h\right)=2^{\sigma(\mathbf{k})},
$$

where $\sigma(\mathbf{k})=k_{1}+k_{2}+k_{3}+\cdots$.

Denote the modular function on $H_{n}$ by $\Delta_{n}$. For $p \leq n$ a similar argument to the one given above shows that

$$
\alpha_{p}^{-1} K_{n} \alpha_{p}=\alpha_{p}\left(K_{n}\right)=K_{n} \cup\left(h^{(p)}+K_{n}\right),
$$

so that $\Delta_{n}\left(\alpha_{p}\right)=2$. However, since $\alpha_{n+1}$ belongs to $Z\left(H_{n}\right)$, it follows that $\Delta_{n}\left(\alpha_{n+1}\right)=1$. Hence $\Delta_{n}$ is not equal to the restriction of $\Delta$ to $H_{n}$ for any $n$, and hypothesis (P) fails in this example. For $\alpha^{\mathbf{k}} h$ in $H_{n}$ we have

$$
\Delta_{n}\left(\alpha^{\mathbf{k}} h\right)=2^{\sigma_{n}(\mathbf{k})},
$$

where $\sigma_{n}(\mathbf{k})=k_{1}+k_{2}+k_{3}+\cdots+k_{n}=\sigma(\mathbf{k})-k_{n+1}$.

\section{A Counterexample to the Extension of Jessen's Theorem.}

In order to show that Jessen's Theorem cannot be extended to the group $G$ it suffices to exhibit a function $f_{1}$ in $L_{1}(G, \lambda)$ such that

$$
\sup _{n} \int_{H_{n}}{ }_{x} f_{1} d \lambda_{n}=\infty
$$

for all $x$ in $G$, where the measures $\lambda_{n}$ are normalised as in $[\mathbf{R} \& \mathbf{S}]$. This will show that Lemma 3 in $[\mathbf{R} \& \mathbf{S}]$ also fails in this example. In fact, we show that such a function $f_{1}$ exists whenever the hypothesis $(\mathrm{P})$ fails. Thus we suppose only that

$$
\left.\Delta\right|_{H_{n}} \neq \Delta_{n} \quad \text { for infinitely many } n \text {. }
$$

To obtain (8), we lose no generality in assuming that

$$
\left.\Delta\right|_{H_{n}} \neq \Delta_{n} \text { for all } n \text {. }
$$

Let $f_{0}$ be any nonzero continuous function with compact support satisfying $0 \leq f_{0} \leq 1$ on $G$. Let $V$ be any symmetric neighbourhood of the identity 
having compact closure, and let $W=V \cdot \operatorname{supp}\left(f_{0}\right)$. Observe that $W$ has compact closure and that

$$
0 \leq f_{0} \leq{ }_{v} \mathbb{I}_{W} \text { for all } v \text { in } W,
$$

where $\mathbb{I}_{W}$ denotes the characteristic function of $W$.

For each $n, \Delta_{n} / \Delta$ is a nontrivial homomorphism of $H_{n}$ into the group of positive real numbers. Thus there exists $y_{n}$ in $H_{n}$ so that $\Delta_{n}\left(y_{n}\right)>4^{n} \cdot \Delta\left(y_{n}\right)$. Let $B_{n}=W y_{n}$ and define

$$
f_{1}=\sum_{k=1}^{\infty} 2^{-k} \Delta\left(y_{k}\right)^{-1} \mathbb{I}_{B_{k}} .
$$

Then $f_{1}$ is a non-negative function and

$$
\begin{aligned}
\int_{G} f_{1} d \lambda & =\sum_{k=1}^{\infty} 2^{-k} \Delta\left(y_{k}\right)^{-1} \lambda\left(B_{k}\right)=\sum_{k=1}^{\infty} 2^{-k} \Delta\left(y_{k}\right)^{-1} \lambda(W) \Delta\left(y_{k}\right) \\
& =\lambda(W)<\infty
\end{aligned}
$$

Hence $f_{1}$ belongs to $L^{1}(G, \lambda)$.

To see that (8) holds, consider $x$ in $G$. Since $G \bigcup_{n=1}^{\infty} V H_{n}$, we can write $x=v z$ where $v$ is in $V$ and $z$ is in $H_{n}$ for sufficiently large $n$. For all $n$, we have

$$
\int_{H_{n}}{ }_{x} f_{1}(y) d \lambda_{n}(y) \geq 2^{-n} \Delta\left(y_{n}\right)^{-1} \int_{H_{n}} \mathbb{I}_{B_{n}}(x y) d \lambda_{n}(y) .
$$

For large $n$, by the invariance of $\lambda_{n}$ under left translation by $z$, we have

$$
\int_{H_{n}} \mathbb{I}_{B_{n}}(x y) d \lambda_{n}(y)=\int_{H_{n}} \mathbb{I}_{B_{n}}(v z y) d \lambda_{n}(y)=\int_{H_{n}} \mathbb{I}_{B_{n}}(v y) d \lambda_{n}(y) .
$$

For $y$ in $H_{n}$ we have by (10)

$$
\mathbb{I}_{B_{n}}(v y)=\mathbb{I}_{W y_{n}}(v y)={ }_{v} \mathbb{I}_{W}\left(y y_{n}^{-1}\right) \geq f_{0}\left(y y_{n}^{-1}\right) .
$$

Therefore for large $n$

$$
\int_{H_{n}} \mathbb{I}_{B_{n}}(x y) d \lambda_{n}(y) \geq \int_{H_{n}} f_{0}\left(y y_{n}^{-1}\right) d \lambda_{n}(y)=\Delta_{n}\left(y_{n}\right) \int_{H_{n}} f_{0} d \lambda_{n}
$$

and

$$
\int_{H_{n}}{ }_{x} f_{1}(y) d \lambda_{n}(y) \geq 2^{-n} \Delta\left(y_{n}\right)^{-1} \Delta_{n}\left(y_{n}\right) \int_{H_{n}} f_{0} d \lambda_{n}>2^{n} \int_{H_{n}} f_{0} d \lambda_{n} .
$$


Since $\lim _{n} \int_{H_{n}} f_{0} d \lambda_{n}=\int_{G} f_{0} d \lambda>0$, we see that (8) holds.

Thus Jessen's Theorem does not extend to any locally compact group $G$, and subgroups $H_{n}$, that do not satisfy hypothesis $(\mathrm{P})$. In the example, one can take $f_{0}=\mathbb{I}_{K}$ and $V=K$, so that $W=K$.

\section{References}

[E\&H] R.E. Edwards and E. Hewitt, Pointwise limits for sequences of convolution operators, Acta Math., 113 (1965), 181-218.

[J] B. Jessen, On the approximation of Lebesgue integrals by Riemann sums, Ann. of Math., 35 (1934), 248-251.

[R\&S] K.A. Ross and K. Stromberg, Jessen's Theorem on Riemann sums for locally compact groups, Pacific J. of Math., 20 (1967), 135-146.

[W1] G. Willis, The structure of totally disconnected, locally compact groups, Math. Ann., 300 (1994), 341-363.

[W2] _ Further properties of the scale function of a totally disconnected, locally compact group, to appear.

Received March 27, 1996.

UNIVERSITY OF OREGON

Eugene, OR 97403-1222

E-mail address: ross@math.uoregon.edu

AND

UNIVERSITY OF NEWCASTLE

CALlaghan, NSW 2308

E-mail address: george@frey.newcastle.edu.au 